\title{
Promising Antibiofilm Activity of Peptidomimetics
}

\author{
Rafael Gomes Von Borowski ${ }^{1,2}$, Simone Cristina Baggio Gnoatto ${ }^{2}$, \\ Alexandre José Macedo ${ }^{2 *}$ and Reynald Gillet ${ }^{1 *}$ \\ ' Univ Rennes, CNRS, Institut de Génétique et Développement de Rennes (IGDR), UMR 6290, Rennes, France, ${ }^{2}$ Programa \\ de Pós-Graduação em Ciências Farmacêuticas, Faculdade de Farmácia, Biotechnology Center, Universidade Federal do Rio \\ Grande do Sul, Porto Alegre, Brazil
}

\section{OPEN ACCESS}

Edited by:

Manuel Simões,

Faculdade de Engenharia,

Universidade do Porto, Portugal

Reviewed by:

César de la Fuente,

Massachusetts Institute of

Technology, United States

Qi Zhao,

University of Dundee, United Kingdom

*Correspondence:

Alexandre José Macedo alexandre.macedo@ufrgs.br

Reynald Gillet

reynald.gillet@univ-rennes1.fr

Specialty section:

This article was submitted to Microbial Physiology and Metabolism,

a section of the journal

Frontiers in Microbiology

Received: 07 June 2018

Accepted: 23 August 2018

Published: 13 September 2018

Citation:

Gomes Von Borowski $R$ Gnoatto SCB, Macedo AJ and Gillet $R$ (2018) Promising Antibiofilm Activity of Peptidomimetics.

Front. Microbiol. 9:2157. doi: 10.3389/fmicb.2018.02157
Pathogenic biofilms are a global health care concern, as they can cause extensive antibiotic resistance, morbidity, mortality, and thereby substantial economic loss. Scientific efforts have been made over the past few decades, but so far there is no effective treatment targeting the bacteria in biofilms. Antimicrobial peptidomimetics have been proposed as promising potential anti-biofilm agents. Indeed, these structurally enhanced molecules can mimic the action of peptides but are not susceptible to proteolysis or immunogenicity, the characteristic limitations of natural peptides. Here, we provide insights into antibiofilm peptidomimetic strategies and molecular targets, and discuss the design of two major peptidomimetics classes: AApeptides ( $N$-acylated- $N$-aminoethyl-substituted peptides) and peptoids ( $N$-substituted glycine units). In particular, we present details of their structural diversity and discuss the possible improvements that can be implemented in order to develop antibiofilm drug alternatives.

Keywords: antibiotic resistance, biofilm, peptides, peptidomimetics, AApeptides, peptoids

\section{INTRODUCTION}

The increased resistance of biofilms to antibiotics is a global health care problem (Costerton et al., 1999; Hall and Mah, 2017). Biofilms are well-organized microbial clusters which produce a matrix from a series of compounds that include extracellular DNA (eDNA), proteins, and polysaccharides. These compounds are either attached to a surface (when originating on medical devices or teeth) or are suspended (in mucus or in chronic wounds) (Flemming and Wingender, 2010). Their form confers advantages over planktonic cells to the matrix-enclosed microorganisms, including improved biocide tolerance, host immune defense, and persistence. These advantages are caused by vast physiological and biochemical changes, including slow cell growth, beneficial quorum sensing, and higher mutation rates (Davies, 2003). Indeed, chronic bacterial infections are themselves encouraged by the accumulation of bacteria in the biofilm-producing biopolymer matrix. Since they are embedded into the matrix, these bacteria have an increased tolerance to antibiotics, chemical disinfectants, and/or host defenses, and are much harder to treat than infections without biofilm (Høiby et al., 2010; Beloin et al., 2014).

The most relevant clinical biofilm-forming bacteria are the gram-negative Acinetobacter baumannii, Escherichia coli, Klebsiella pneumoniae, and Pseudomonas aeruginosa, along with grampositive Staphylococcus aureus and the less virulent S. epidermidis (Jabbouri and Sadovskaya, 2010; de la Fuente-Núñez et al., 2013; Chen et al., 2014; Culotti and Packman, 2014; Longo et al., 2014; Andrea et al., 2018). These microorganisms can form biofilms on virtually any medical device, including cardiac pacemakers and prosthetic heart valves, endotracheal tubes, urinary catheters, 
central venous catheters, prostheses, orthopedic devices, contact lenses, and dentures (Baquero and Coque, 2011). This ability is possible due the broad genetic variability of the microbial populations found in health care institutions. This genetic spectrum, also implying phenotypic variations, occurs within the same species. This makes it difficult to develop a therapy or even a general surface material that could deter the growth and adhesion of these microorganisms (Cegelski et al., 2008). Medical devices are an important cause of human infections, for instance turning $S$. epidermidis into an important emerging pathogen responsible for most infections in central venous catheters. This results in the need to remove and replace the medical device, increasing costs and patient suffering (Maki et al., 2006). Not only do bacteria have the individual capacity to form biofilm, but in biofilm some strains will have increase their horizontal transfers of plasmids carrying antibiotic resistance genes, thus increasing mutation frequency (Savage et al., 2013). For all of these reasons, pathogenic biofilms have a huge clinical impact in terms of economic losses, morbidity, and mortality.

Therefore, bacterial biofilms are promising targets for combatting this problem of antibiotic resistance. The successful development of antibiofilm compounds will therefore be an important tool for controlling human infections (Miquel et al., 2016).

In this context, peptides have been proposed as an important direction to follow, either for creating alternative drug therapies or for developing new anti-infective surfaces (Riool et al., 2017).

Peptides are fundamental molecules made up of 2-50 amino acids, with many biological functions. Indeed, their versatile chemical features such as malleability and multifunctionality, make them good models for the synthesis of new bioactive compounds (Von Borowski et al., 2017). Antimicrobial peptides (AMPs) are very interesting molecules to be explored in the search for antibiofilm agents to replace conventional antibiotics. This is because they are relatively easy to produce while exhibiting broad-spectrum antimicrobial activity, with a distinct mode of action that means that they are less prone to developing resistance (de la Fuente-Núñez et al., 2013; Strempel et al., 2015; Andrea et al., 2018). However, although natural peptides are indispensable for the structure, functioning, and metabolism of each living organism, their regulation is mediated by molecular interactions, proteolysis, and immunogenic responses (Avan et al., 2014). Thus low stability and availability limits their therapeutic relevance. On the other hand, peptidomimetics are chemically modified expressly to limit the drawbacks of natural peptides. The underlying strategy is to create small peptide-like molecules that still have the inherent abilities of natural ones (so that the advantageous biological effects remain), but which are more stable and available, with improved selectivity and/or potency (Grauer and König, 2009; Croft and Purcell, 2011). In this context, rather than joining the amino acids to a bioisosteric group to mimic the original amide, a very efficient chemical strategy is to replace the peptide bond, the -CO-NH- amide (Niu et al., 2013). Although quite a number of amide bond replacements have been reported, our review focuses here on the $N$-acylated- $N$-aminoethyl amino acids (AApeptides) and on peptoids. These specific strategies were chosen as they each have pronounced chemical diversity, can mimic both the primary and secondary structures of peptides, resist proteolysis, and show good activity against pathogenic biofilms. By presenting these promising candidates, we pave the way for the design of more active and safer innovative molecules.

\section{PEPTIDOMIMETICS ARE AN IMPROVEMENT OVER NATURAL PEPTIDES}

Despite their inherent robust and promising bioactivity, there are drawbacks to the use of natural peptides, including their high clearance and their susceptibility to proteolysis or immunogenicity, both of which can cause unwanted effects (Von Borowski et al., 2017). Inspired by natural peptides, chemists have developed a variety of structurally diverse synthetic mimics with key physicochemical natures (i.e., cationic charges and amphiphilicity) which they call peptidomimetics.

These molecules can be obtained in different ways. Peptidomimetics can be made by manipulating the amino acid backbone of native peptides in order to enrich structural diversity, making them extraordinarily useful. They can also be prepared through the coupling of stable unnatural amino acids generated via modifications such as amine alkylation. For example, poly-N-substituted glycines allow for the generation of peptoids that differ from peptides only in their side chains, making them protease-resistant (Miller et al., 1995). Another strategy is the isosteric replacement of the amino group by for example an oxygen or sulfur atom. This changes the H-bonding pattern, significantly affecting the secondary structure and folding properties of peptides. Another possible approach for getting peptidomimetics that have new secondary structures and biological activities involves taking natural peptides and performing $\mathrm{C}-\alpha$ configuration inversion, $\alpha$-hydrogen replacement (by the alkyl or other groups), and replacing the $\alpha$-carbon atom by heteroatom, mostly nitrogen (Avan et al., 2014). Several of these strategies have been used to synthesize peptidomimetics which appear to be as promising against biofilms as naturally occurring AMPs. In fact, many synthetic antibacterial peptidomimetics are currently undergoing clinical trials, including the membrane-disrupting compound LTX109, the cationic steroid compound CSA-13, and the novel peptidomimetic brilacidin (see https://clinicaltrials.gov/). Recently, short peptidomimetics made of Arg and N-alkyl/aryl pyrazole residues were shown to have good antimicrobial and anti-inflammatory activities, increased proteolytic stability against trypsin digestion, and antimicrobial activity, even in the presence of physiological salts (Ahn et al., 2017). Another series of AMP mimetics were synthesized by incorporating a 3'-amino-[1,1'-biphenyl]-3-carboxylic acid backbone in MSI-78, a peptide currently in Phase-III clinical trials (Kuppusamy et al., 2018).

\section{AApeptides as a Peptidomimetic Strategy}

AApeptides are oligomers of $N$-acylated- $N$-aminoethylsubstituted amino acids that are derived from chiral peptide 


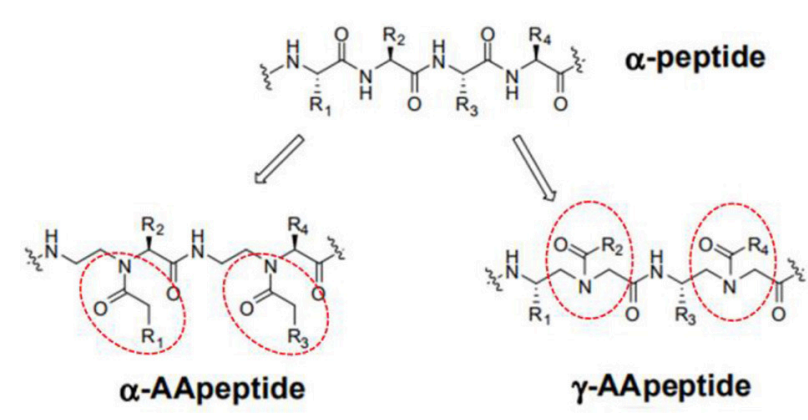

FIGURE 1 | Structure illustration of an $\alpha$-peptide and its corresponding AApeptide models, adapted from Niu et al. (2013). The $\alpha$ - and $\gamma$ $N$-acylated- $N$-aminoethyl amino acid amide bond replacement structures are identified by dotted circles in red. $\mathrm{R}$ corresponds to possible radicals.

nucleic acid (PNA) backbones (Shi et al., 2016). The chiral side chain is connected to either the $\alpha-\mathrm{C}$ or $\gamma$-C of the carbonyl group, while acylation is used to introduce the other side chain to the central N, as illustrated in Figure 1 (Sang et al., 2017). Compared to their original peptide counterparts, AApeptides have the same backbone lengths and functional group counts, and the same number of nitrogen atoms involved in secondary or tertiary amide bonds. In addition, they mimic the original amino acid side-chain positions, so they have the same activity. However, their backbones are more flexible, and since the AApeptides have tertiary amide bonds that can be involved in cis/trans configurations, they should have interesting hydrogen bonding properties and conformational flexibilities (Niu et al., 2013).

\section{Antibiofilm AApeptides}

In a recent study, Teng et al. (2016) described an acyclic model based on host defense peptides (HDPs) with charged and non-charged radicals (Table 1, ID 1). The global structure has a cationic hydrophobic residue composed of ornithine, and adamantyl or aromatic rings. This model was used to produce a global amphiphilic AApeptide that targets membrane disruption for antibiotic activity. The molecule displays high Gram-negative antifouling activity and low cytotoxicity effects. In addition, it was hypothesized that if radical 1 (R1) was a cationic group, and R2, R3, and R4 were hydrophobic, the global structure should be both an HDP and amphipathic, which will result in the killing of bacteria via membrane disruption. Accordingly, the hydrophobicities of R2, R3, and R4 were modified by inserting various groups (such as adamantyl, biphenyl, CF3, t-butyl) into the aromatic rings, then testing the resulting compounds against clinically relevant bacteria. A reduction in R2-R4 hydrophobicity correlates to decreased molecule killing capacities.

In order to verify the selectivity of the compounds, cationic residues such as lysine, ornithine, and arginine were added, and the hemolysis profiles assessed. Lysine decreases hemolytic and antibacterial activities, ornithine increases them, and arginine has no effect. Often amphipathic agents are cytotoxic, but at a concentration of $25 \mu \mathrm{g} / \mathrm{mL}$, the compounds did not show noticeable cytotoxicity against either the HK-2 renal epithelial cell line or the K562 human erythroleukemic one. Table 1 details the most promising AApeptide, "Compound 13," which was tested for antibiofilm activity at concentrations below the minimal inhibitory concentration (MIC). At this level, a 50-75\% reduction of biofilm formation was seen via crystal violet staining in both E. coli (ATCC 25922) and A. baumannii.

In another study, Padhee et al. (2015) assessed the biofilm antifouling and eradication activities of peptidomimetics based on the structures of daptomycin and polymyxin $\mathrm{B}$. The main compound, YL-36, is a cyclic $\gamma$-AApeptide having both charged and neutral radicals (Table 1, ID 2). YL-36 was designed by joining lipid tails from amphiphilic building blocks with the cyclic rings, with ornithine as cationic residues. Lipo-cyclic structures turn out to have a broader-spectrum of antimicrobial activity than the others, and they work against inflammation by suppressing pro-inflammatory cytokines. With YL-36, 70-80\% biofilm antifouling activity was observed with both $P$. aeruginosa and Methicillin-resistant Staphylococcus epidermidis (MRSE), although these results are inconclusive since the concentrations tested were over the MIC. In any case, the potential for antibiofilm activity could be due to the presence of lipid tails which can retard biofilm formation. The structures that are globally amphipathic probably line up to form micelles when there is an interaction with the polyanionic exopolysaccharide matrix, ultimately disrupting it. Note that YL-36 is not hemolytic, meaning that this compound is highly selective.

\section{Peptoids as a Peptidomimetic Strategy}

Peptoids are oligomers of $N$-substituted glycine units (Figure 2). Their side chains extend from the main-chain nitrogen rather than from the $\alpha$-carbon, thus yielding secondary structures including helices, loops, and turns. They are achiral foldamer molecules, and retain the functionalities and backbone polarity of peptides (Yoo and Kirshenbaum, 2008; Zuckermann and Kodadek, 2009; Mándity and Fülöp, 2015).

\section{Antibiofilm Peptoids}

Hoque et al. (2015) explored a series of small acyclic amphiphilic peptoids based on AMP structures. They did this by inserting two non-amino acid positive charges, two lipophilic alkyl moieties, and two non-peptidic amide groups (Table 1, ID 3). They demonstrated that antimicrobial activity and hemolytic action correlate to the lipophilic alkyl chain/spacer and increases in chain length, which changes selectivity. The most promising molecule, "Compound 2d" (Table 2, ID 3), shows optimum amphiphilicity, and is able to disperse both $S$. aureus and E. coli mature biofilms at the solid-liquid and liquid-air interfaces, with complete eradication at $32 \mu \mathrm{g} / \mathrm{mL}$ even while biofilm is already formed on the cover slips. In addition, $2 \mathrm{~d}$ also decreases bacterial viability inside the biofilm, whereas the cell viability of nontreated biofilm increases. Although the study made no mention of an antibiofilm structure-activity relationship (SAR), 2d was non-toxic to human erythrocytes and human kidney cells.

Kapoor et al. (2011) selected several peptoid analogs to an acyclic amphipathic and cationic dodecamer peptoid (Table 2, ID 4) based on AMP structures. The alkylated peptoids were 


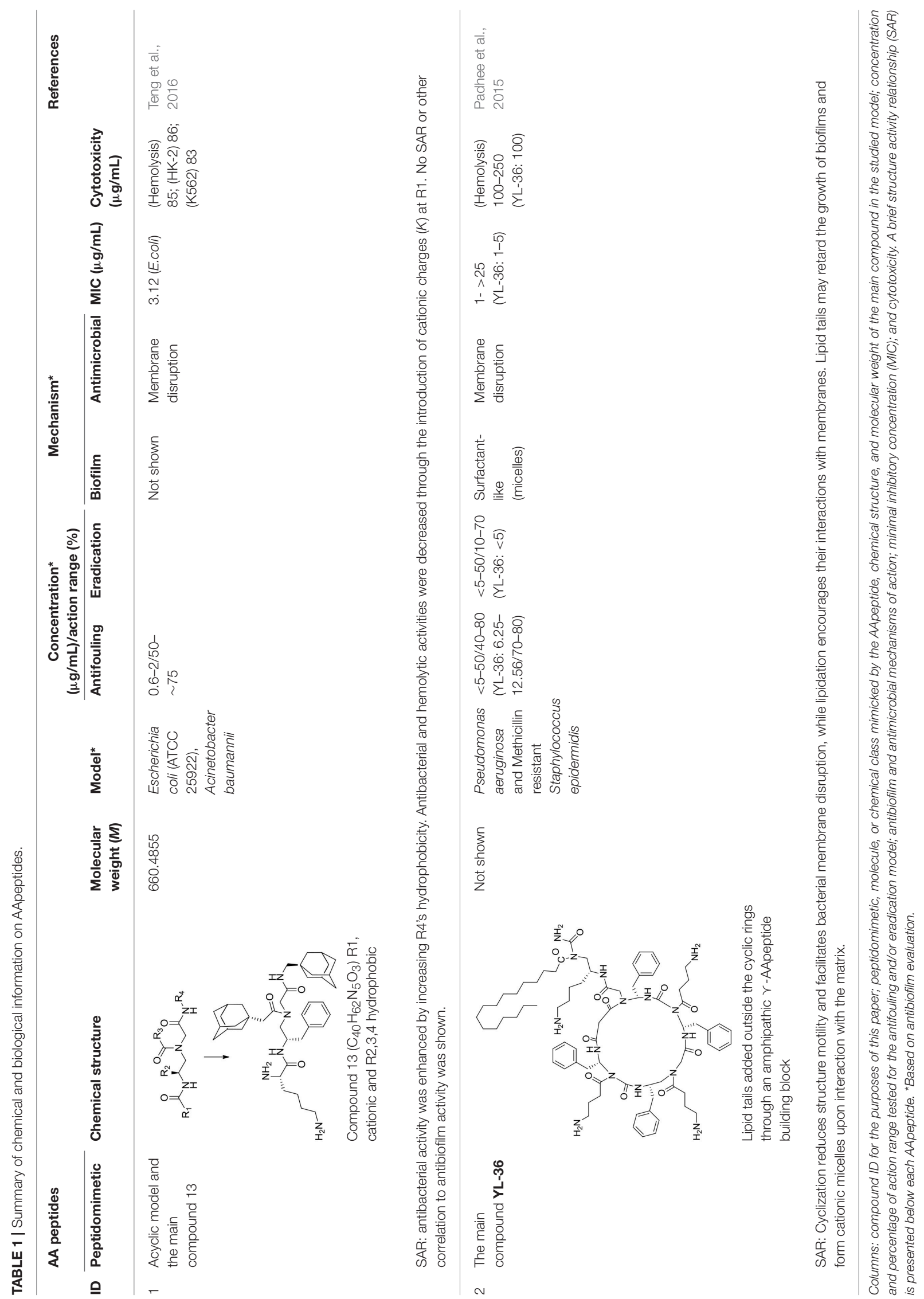



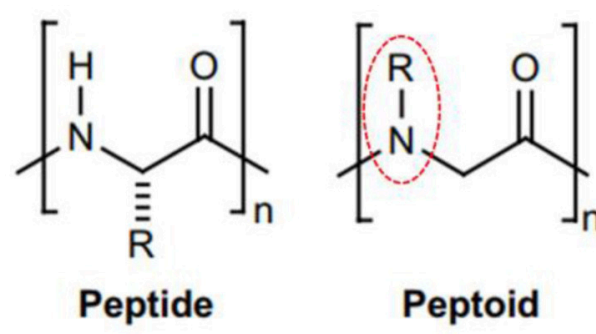

FIGURE 2 | Peptide and peptoid monomer structures differ. (Left) Illustration of a classic glycine peptide unit, which has a chiral carbon linked to amino, carboxyl, and radical groups. (Right) An $\mathrm{N}$-substituted $\mathrm{R}=\mathrm{H}$ for glycine amino acids residues. This has a radical group linked to the amino group instead of the chiral carbon, identified by dotted circles in red.

active against planktonic cells, while the unalkylated ones were not. The $1-\mathrm{C} 13_{4 \text { mer }}$ peptoid (H-Ntridec-NLys-Nspe-Nspe-NLys$\mathrm{NH}_{2}$ ) is the main compound, preventing about $70 \%$ of biomass formation in P. aeruginosa. In addition, peptoids 1 and $1-\mathrm{C} 13_{4 \mathrm{mer}}$ impaired preformed biofilms by 60 and $40 \%$, and reduced cell viability by about 1.5 and 3 logs, respectively. Antibiofilm activity against $P$. aeruginosa was measured at $12.5 \mu \mathrm{g} / \mathrm{mL}$ (MIC) and the biomass was assessed with crystal violet staining. Still, the authors discuss the possibility of using peptoids to bind DNA and facilitate biofilm detachment and/or disruption. Indeed, their activity could be due to their inherent oligomerization via aromatic side-chain interactions. The hydrophobic tails might bestow their efficiency in reducing cell viability, as these confer a surfactant-like nature which causes micelles to form. Micelles may strongly interact with and disrupt the hydrophobic exopolysaccharide matrix, facilitating deeper peptoid penetration into the matrix.

Konai and Haldar (2015) went in the opposite direction. They started with spermidine and norspermidine polyamine structures, both known to have antibiofilm properties. Looking for significant antibacterial activity at minimum biofilm inhibitory concentrations (MBICs), they synthesized a series of acyclic amphipathic conjugate molecules (Table 2, ID 5). These contained a cationic moiety made of various fatty acids acting as lipophilic tails and the amino acid lysine (L-lysine and D-lysine), a known trigger for biofilm disassembly. Crystal violet staining, confocal imaging, and killing curve determination showed that "Compound 8" reduces S. aureus viability in preformed biofilm in a concentration-dependent manner. However, the best MBIC value $(116 \mu \mathrm{M})$ was found in derivatives " 4 " (L,L configuration, $\left.R=\mathrm{C}_{15} \mathrm{H}_{31}\right)$ and "9" (D,D configuration, $\left.R=\mathrm{C}_{15} \mathrm{H}_{31}\right)$. The mechanism of biofilm disruption is still being investigated, but improved electrostatic and hydrogen bonding interactions with the biofilm's extracellular matrix components may play a major role. The compounds were non-toxic to erythrocytes.

Yang Liu et al. (2013) previously described a synthetic approach to designing acyclic oligomers based on AMP structures with alternating repeats of $\alpha$-amino acids and $\beta$-peptoid residues. The representative compounds for two subclasses are shown in Table 2 (ID 6). These have chain lengths of 4-16 residues, and longer chain lengths often correlated with increased antimicrobial activity within the subclass. This tendency was more pronounced in the lysinecontaining groups ( 1 and 4 ) than in the homoarginine-rich ones (2 and 3). The hybrid oligomers also inhibited S. epidermidis biofilm formation and displayed antibiofilm activity against preformed S. epidermidis biofilm. In comparison with their lysine-containing counterparts (such as $1 \mathrm{~d}$ and $4 \mathrm{c}$ ), the fully guanidinylated (e.g., hArg-rich) oligomers (e.g., "2b”) killed slowgrowing cells faster, and had more antibiofilm capacity. Chirality appears to be essential for the efficient killing of both slowgrowing planktonic cells and biofilms in all the studied oligomers. They were not toxic to erythrocytes, but are toxic to HeLa cells in a concentration-dependent manner. To keep cytotoxicity at acceptable levels, a promising strategy may be to design alternating oligomers that display only a 1:1 ratio of amino or guanidino/amino functional groups.

Finally, since the antibiotic bioactivity that has been explored is usually due to membrane disruption, cationic molecules and bacterial membrane structure-activity relationships have been thoroughly investigated, and amphiphilic molecules probably act in the same way since they act as partial cationics. Moreover, the evaluation of cytotoxicity levels shows that both peptidomimetics and their expected amphiphilicities have good potentials. Finally, both AApeptides and peptoids have been shown to act as effective antibiofilm agents, although their bioactivity and selectivity depend on optimal amphiphilicity. Therefore, we highlight that acyclic conformation and lipid tails, neutral aromatic compounds, and ornithine substituents should be the most advantageous peptidomimetic structural improvements in order to obtain antibiofilm molecules.

\section{CONCLUSION}

In microorganisms, biofilm lifestyle is a significant virulence factor that results in enhanced resistance to medical treatment (Otto, 2014). This means that antibiotics are less effective, and clearly biofilms have a considerable clinical impact (Del Pozo, 2018). Methods for combatting biofilms using natural peptides seem promising, but their therapeutic relevance is limited by inherently low stability and availability (Kang et al., 2014). Therefore, antibiofilm peptidomimetics are being studied as way to mimic natural peptides while avoiding their drawbacks (Mizuno et al., 2017).

In short, the active structures we discuss tend to mimic naturally occurring antimicrobial molecules such as host defense peptides (HDPs) and antimicrobial peptides (AMPs). These are both endogenous polypeptides produced by multicellular organisms, and they act as an evolutionarily conserved mechanism of innate immune defense (Huang et al., 2014). Thousands of these peptides have been identified in bacteria, plants, insects, birds, fish, and mammals. Family members are highly diverse in their sequences, but generally of small size, made up of 12-50 amino acids. They have similar overall cationic charges of +2 to +9 , and are amphipathic, 

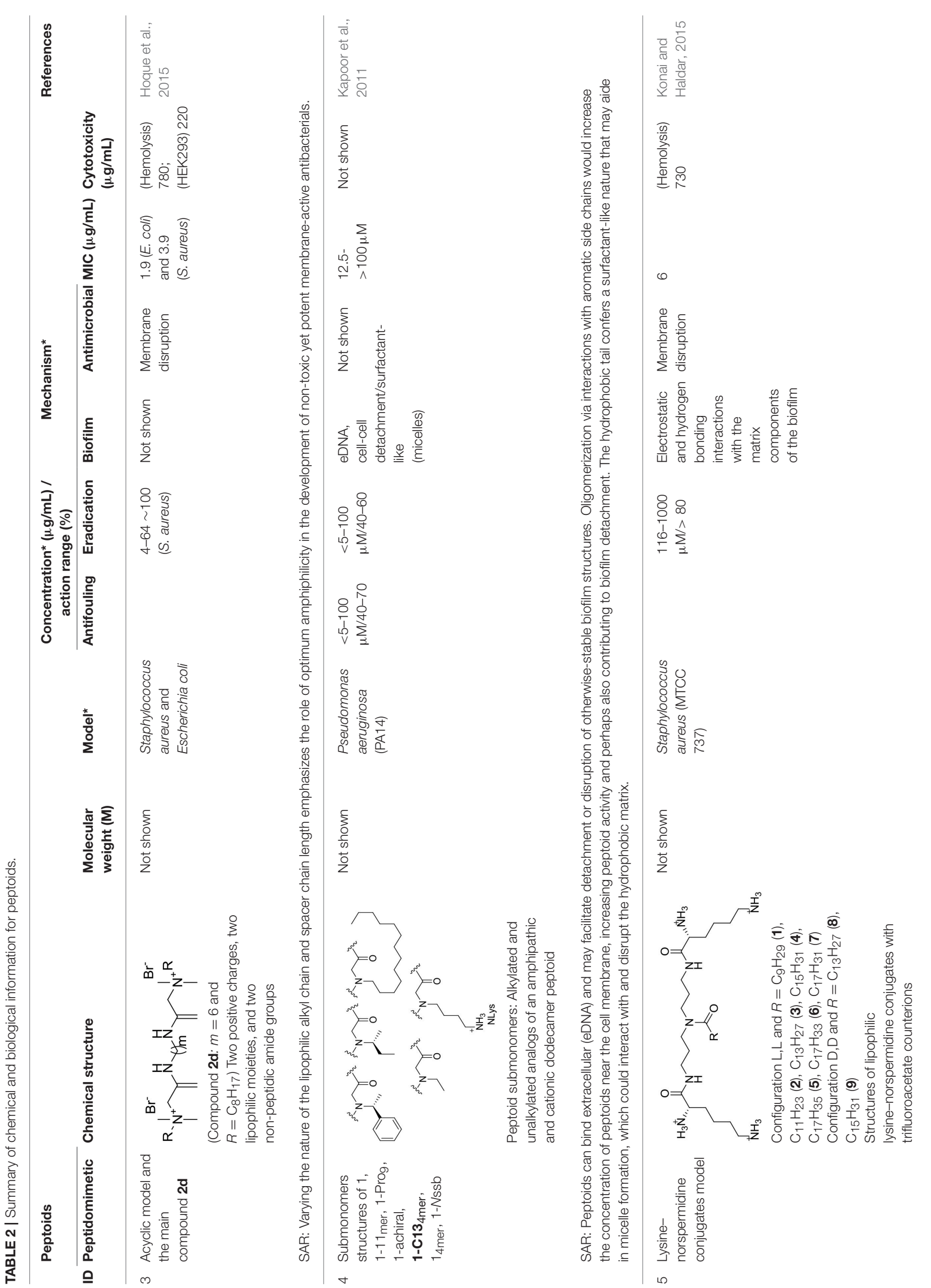


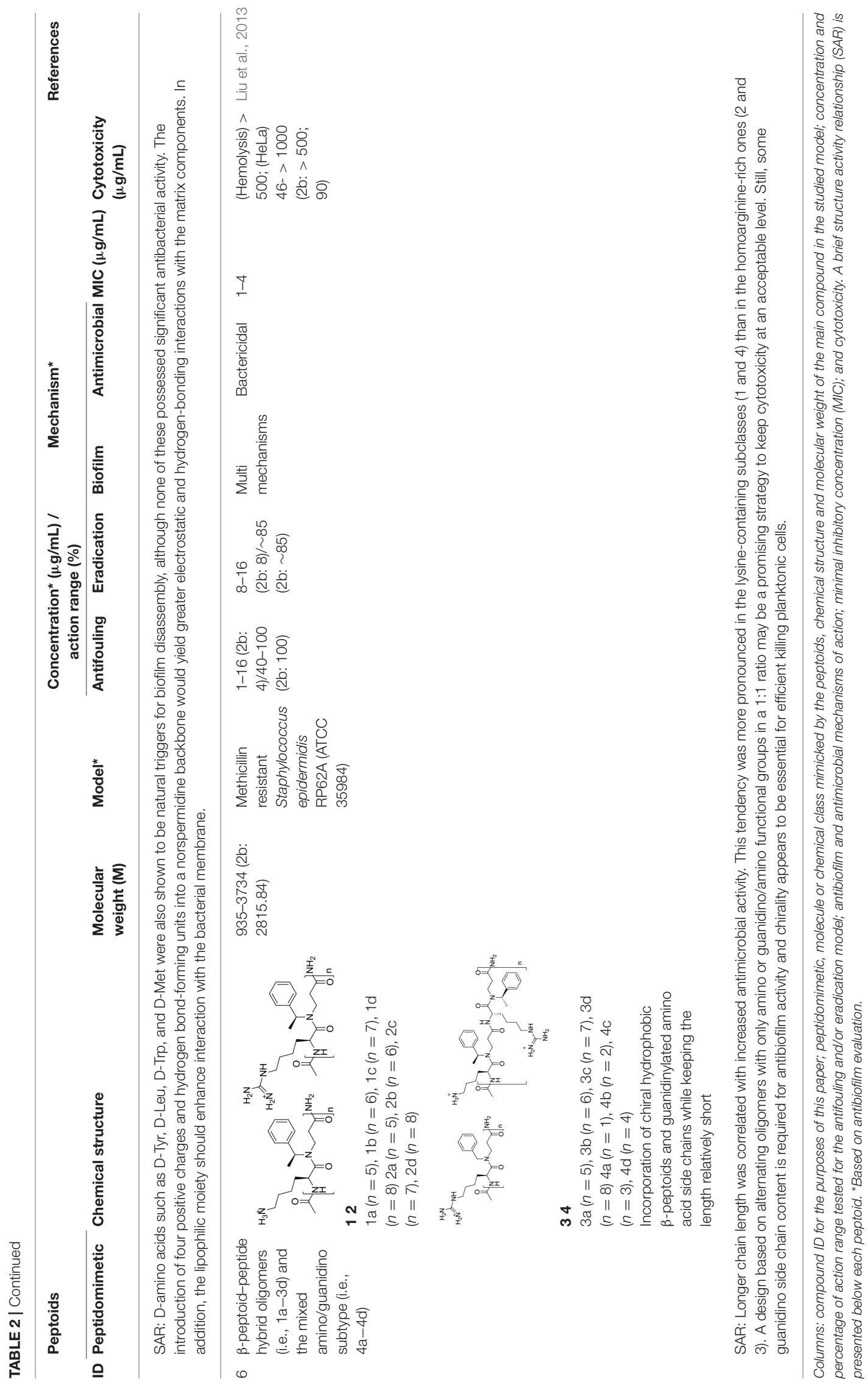


with over 50\% hydrophobic residues (Kindrachuk and Napper, 2010). Moreover, it is known that AMPs show broad-spectrum antimicrobial activities and have a low propensity for developing resistance (Seo et al., 2012; Lázár et al., 2018). Peptidomimetics are the same, even though they are all designed to be amphiphilic instead of cationic like AMPs. To confer the desired amphiphilicity, the molecules are linked to different substituents, which are charged and non-charged radicals. The most relevant of those are lipid tails, neutral aromatic compounds, and charged ornithine amino acids.

Although there is an incomplete understanding of the mechanisms of action of antibiofilm peptidomimetics, it seems that their amphiphilicity improves their hydrogen and electrostatic interactions with matrix components, such as those with surfactants.

In the past 40 years, more than 30,000 articles have been published about microbial biofilms (source: PubMed database). This mass of research has been dedicated to understanding biofilms dynamics and to decreasing its effects, but so far no effective treatment has been developed (Bjarnsholt et al., 2013). Antibiofilm AApeptides and peptoids are two very promising families of peptidomimetics for the development and refining of new antibiofilm agents to be used in the fight against resistant microorganisms.

\section{REFERENCES}

Ahn, M., Gunasekaran, P., Rajasekaran, G., Kim, E. Y., Lee, S. J., and Bang, G. (2017). Pyrazole derived ultra-short antimicrobial peptidomimetics with potent anti-biofilm activity. Eur. J. Med. Chem. 125, 551-564. doi: 10.1016/j.ejmech.2016.09.071

Andrea, A., Molchanova, N., and Jenssen, H. (2018). Antibiofilm peptides and peptidomimetics with focus on surface immobilization. Biomolecules 8:E27. doi: 10.3390/biom 8020027

Avan, I., Hall, C. D., and Katritzky, A. R. (2014). Peptidomimetics via modifications of amino acids and peptide bonds. Chem. Soc. Rev. 43, 3575-3594. doi: 10.1039/c3cs60384a

Baquero, F., and Coque, T. M. (2011). Multilevel population genetics in antibiotic resistance. FEMS Microbiol. Rev. 35, 705-706. doi: 10.1111/j.1574-6976.2011.00293.x

Beloin, C., Renard, S., Ghigo, J. M., and Lebeaux, D. (2014). Novel approaches to combat bacterial biofilms. Curr. Opin. Pharmacol. 18, 61-68. doi: 10.1016/j.coph.2014.09.005

Bjarnsholt, T., Ciofu, O., Molin, S., Givskov, M., and Høiby, N. (2013). Applying insights from biofilm biology to drug development - can a new approach be developed? Nat Rev. Drug Discov. 12, 791-808. doi: 10.1038/ nrd4000

Cegelski, L., Marshall, G. R., Eldridge, G. R., and Hultgren, S. J. (2008). The biology and future prospects of antivirulence therapies. Nat. Rev. Microbiol. 6, 17-27. doi: 10.1038/nrmicro1818

Chen, P., Seth, A. K., Abercrombie, J. J., Mustoe, T. A., and Leung, K. P. (2014). Activity of imipenem against Klebsiella pneumoniae biofilms in vitro and in vivo. Antimicrob. Agents Chemother. 58, 1208-1213. doi: 10.1128/AAC.01353-13

Costerton, J. W., Stewart, P. S., and Greenberg, E. P. (1999). Bacterial biofilms: a common cause of persistent infections. Science 284, 1318-1322. doi: $10.1126 /$ science.284.5418.1318

Croft, N. P., and Purcell, A. W. (2011). Peptidomimetics: modifying peptides in the pursuit of better vaccines. Expert Rev. Vaccines 10, 211-226. doi: $10.1586 /$ erv. 10.161

\section{AUTHOR CONTRIBUTIONS}

RG wrote the manuscript with support from SG, AM, and RGB. All authors provided critical feedback and helped shape the manuscript.

\section{FUNDING}

This study was funded by the CAPES-COFECUB program. The institutional partners of this partnership between Brazil and France are the Brazilian Ministry of Education's CAPES (Coordenação de Aperfeiçoamento de Pessoal de Nível Superior) agency, and the French Ministère de l'Europe et des Affaires étrangères (MEAE) and the Ministère de l'Enseignement supérieur, de la Recherche et de l'Innovation (MESRI). This work was also supported by the French Agence Nationale pour la Recherche and Direction Générale de l'Armement (\#ANR-14ASTR-0001).

\section{ACKNOWLEDGMENTS}

We thank all of the people involved in the CAPES-COFECUB project as well as Juliana Berland for insightful comments on the manuscript.

Culotti, A., and Packman, A. I. (2014). Pseudomonas aeruginosa promotes Escherichia coli biofilm formation in nutrient-limited medium. PLoS ONE 9:e107186. doi: 10.1371/journal.pone.0107186

Davies, D. (2003). Understanding biofilm resistance to antibacterial agents. Nat. Rev. Drug Discov. 2, 114-122. doi: 10.1038/nrd1008

de la Fuente-Núñez, C., Reffuveille, F., Fernández, L., and Hancock, R. E. (2013). Bacterial biofilm development as a multicellular adaptation: antibiotic resistance and new therapeutic strategies. Curr. Opin. Microbiol. 16, 580-589. doi: 10.1016/j.mib.2013.06.013

Del Pozo, J. L. (2018). Biofilm-related disease. Expert Rev. Anti Infect. Ther. 16, 51-65. doi: 10.1080/14787210.2018.1417036

Flemming, H.-C., and Wingender, J. (2010). The biofilm matrix. Nat. Rev. Microbiol. 8, 623-633. doi: 10.1038/nrmicro2415

Grauer, A., and König, B. (2009). Peptidomimetics - a versatile route to biologically active compounds. Eur. J. Org. Chem. 30, 5099-5111. doi: 10.1002/ejoc.200900599

Hall, C. W., and Mah, T. F. (2017). Molecular mechanisms of biofilm-based antibiotic resistance and tolerance in pathogenic bacteria. FEMS Microbiol. Rev. 41, 276-301. doi: 10.1093/femsre/fux010

Høiby, N., Bjarnsholt, T., Givskov, M., Molin, S., and Ciofu, O. (2010). Antibiotic resistance of bacterial biofilms. Int. J. Antimicrob. Agents 35, 322-332. doi: 10.1016/j.ijantimicag.2009.12.011

Hoque, J., Konai, M. M., Samaddar, S., Gonuguntala, S., Manjunath, G. B., and Ghosh, C., et al. (2015). Selective and broad spectrum amphiphilic small molecules to combat bacterial resistance and eradicate biofilms. Chem. Commun. 51, 13670-13673. doi: 10.1039/C5CC05159B

Huang, W., Seo, J., Willingham, S. B., Czyzewski, A. M., Gonzalgo, M. L., and Weissman, I. L. (2014). Learning from host-defense peptides: cationic, amphipathic peptoids with potent anticancer activity. PLoS ONE 9:e90397. doi: 10.1371/journal.pone.0090397

Jabbouri, S., and Sadovskaya, I. (2010). Characteristics of the biofilm matrix and its role as a possible target for the detection and eradication of Staphylococcus epidermidis associated with medical implant infections. FEMS Immunol. Med. Microbiol. 59, 280-291. doi: 10.1111/j.1574-695X.2010. 00695.x 
Kang, S. J., Park, S. J., Mishig-Ochir, T., and Lee, B. J. (2014). Antimicrobial peptides: therapeutic potentials. Expert Rev. Anti Infect. Ther. 12, 1477-1486. doi: $10.1586 / 14787210.2014 .976613$

Kapoor, R., Wadman, M. W., Dohm, M. T., Czyzewski, A. M., Spormann, A. M., and Barron, A. E. (2011). Antimicrobial peptoids are effective against Pseudomonas aeruginosa biofilms. Antimicrob. Agents Chemother. 55, 3054-3057. doi: 10.1128/AAC.01516-10

Kindrachuk, J., and Napper, S. (2010). Structure-activity relationships of multifunctional host defence peptides. Mini Rev. Med. Chem. 10, 596-614. doi: $10.2174 / 138955710791383983$

Konai, M. M., and Haldar, J. (2015). Lysine-based small molecules that disrupt biofilms and kill both actively growing planktonic and nondividing stationary phase bacteria. ACS Infect. Dis. 1, 469-478. doi: 10.1021/acsinfecdis.5b00056

Kuppusamy, R., Yasir, M., Berry, T., Cranfield, C. G., Nizalapur, S., and Yee, E. (2018). Design and synthesis of short amphiphilic cationic peptidomimetics based on biphenyl backbone as antibacterial agents. Eur. J. Med. Chem. 143, 1702-1722. doi: 10.1016/j.ejmech.2017.10.066

Lázár, V., Martins, A., Spohn, R., Daruka, L., Grézal, G., and Fekete, G. (2018). Antibiotic-resistant bacteria show widespread collateral sensitivity to antimicrobial peptides. Nat. Microbiol. 3, 718-731. doi: 10.1038/s41564-018-0164-0

Liu, Y., Knapp, K. M., Yang, L., Molin, S., Franzyk, H., and Folkesson, A. (2013). High in vitro antimicrobial activity of beta-peptoid-peptide hybrid oligomers against planktonic and biofilm cultures of Staphylococcus epidermidis. Int. J. Antimicrob. Agents 41, 20-27. doi: 10.1016/j.ijantimicag.2012.09.014

Longo, F., Vuotto, C., and Donelli, G. (2014). Biofilm formation in Acinetobacter baumannii. New Microbiol. 37, 119-127.

Maki, D. G., Kluger, D. M., and Crnich, C. J. (2006). The risk of bloodstream infection in adults with different intravascular devices: a systematic review of 200 published prospective studies. Mayo Clin. Proc. 81, 1159-1171. doi: $10.4065 / 81.9 .1159$

Mándity, I. M., and Fülöp, F. (2015). An overview of peptide and peptoid foldamers in medicinal chemistry. Expert Opin. Drug Discov. 10, 1163-1177. doi: $10.1517 / 17460441.2015 .1076790$

Miller, S. M., Simon, R. J., Zuckermann, R. J., Kerr, J. M., Moos, W. H., et al. (1995). Comparison of the proteolytic susceptibilities of homologous L-amino acid, Damino acid, and N-substituted glycine peptide and peptoid oligomers. Drug Dev. Res. 35, 20-32.

Miquel, S., Lagrafeuille, R., Souweine, B., and Forestier, C. (2016). Anti-biofilm activity as a health issue. Front. Microbiol. 7:592. doi: 10.3389/fmicb.2016.00592

Mizuno, A., Matsui, K., and Shuto, S. (2017). From peptides to peptidomimetics: a strategy based on the structural features of cyclopropane. Chemistry 23, 14394-14409. doi: 10.1002/chem.201702119

Niu, Y., Wu, H., Li, Y., Hu, Y., Padhee, S., and Li, Q., et al. (2013). AApeptides as a new class of antimicrobial agents. Org. Biomol. Chem. 11, 4283-4290. doi: $10.1039 / \mathrm{c} 3 \mathrm{ob} 40444 \mathrm{~g}$

Otto, M. (2014). Physical stress and bacterial colonization. FEMS Microbiol Rev. 38, 1250-1270. doi: 10.1111/1574-6976.12088
Padhee, S., Li, Y. Q., and Cai, J. F. (2015). Activity of lipo-cyclic gamma-AApeptides against biofilms of Staphylococcus epidermidis and Pseudomonas aeruginosa. Bioorg. Med. Chem. Lett. 25, 2565-2569. doi: 10.1016/j.bmcl.2015.04.039

Riool, M., de Breij, A., Drijfhout, J. W., Nibbering, P. H., and Zaat, S. A. (2017). Antimicrobial peptides in biomedical device manufacturing. Front. Chem. 5:63. doi: 10.3389/fchem.2017.00063

Sang, P., Shi, Y., Teng, P., Cao, A., Xu, H., and Li, Q.,et al. (2017). Antimicrobial AApeptides. Curr. Top. Med. Chem. 17, 1266-1279. doi: $10.2174 / 1568026616666161018145945$

Savage, V. J., Chopra, I., and O'neill, A. J. (2013). Staphylococcus aureus biofilms promote horizontal transfer of antibiotic resistance Antimicrob. Agents Chemother. 57, 1968-1970. doi: 10.1128/AAC.02 008-12

Seo, M. D., Won, H. S., Kim, J. H., Mishig-Ochir, T., and Lee, B. J.,et al. (2012). Antimicrobial peptides for therapeutic applications. Rev. Mol. 17, 12276-12286. doi: $10.3390 /$ molecules 171012276

Shi, Y., Teng, P., Sang, P., She, F., Wei, L., and Cai, J. (2016). gammaAApeptides: design, structure, and applications. Acc. Chem. Res. 49, 428-441. doi: 10.1021/acs.accounts.5b00492

Strempel, N., Strehmel, J., and Overhage, J. (2015). Potential application of antimicrobial peptides in the treatment of bacterial biofilm infections. Curr. Pharma. Design 21, 67-84. doi: 10.2174/13816128206661409051 24312

Teng, P., Huo, D., Nimmagadda, A., Wu, J., She, F., and Su, M. (2016). Small antimicrobial agents based on acylated reduced amide scaffold. J. Med. Chem. 59, 7877-7887. doi: 10.1021/acs.jmedchem.6b00640

Von Borowski, R. G., Macedo, A. J., and Gnoatto, S. C. B. (2017). Peptides as a strategy against biofilm-forming microorganisms: Structureactivity relationship perspectives. Eur. J. Pharm. Sci. 114, 114-137. doi: $10.1016 /$ j.ejps.2017.11.008

Yoo, B., and Kirshenbaum, K. (2008). Peptoid architectures: elaboration, actuation, and application. Curr. Opin. Chem. Biol. 12, 714-721. doi: $10.1016 /$ j.cbpa.2008.08.015

Zuckermann, R. N., and Kodadek, T. (2009). Peptoids as potential therapeutics. Curr. Opin. Mol. Therap. 11, 299-307.

Conflict of Interest Statement: The authors declare that the research was conducted in the absence of any commercial or financial relationships that could be construed as a potential conflict of interest.

Copyright (c) 2018 Gomes Von Borowski, Gnoatto, Macedo and Gillet. This is an open-access article distributed under the terms of the Creative Commons Attribution License (CC BY). The use, distribution or reproduction in other forums is permitted, provided the original author(s) and the copyright owner(s) are credited and that the original publication in this journal is cited, in accordance with accepted academic practice. No use, distribution or reproduction is permitted which does not comply with these terms. 